\title{
A special acute care surgery model in China: How to improve the efficiency of emergency clinical diagnosis and treatment.
}

\section{Dequan Xu}

First Affiliated Hospital of Harbin Medical University

\section{Yue Yin}

First Affiliated Hospital of Harbin Medical University

Limin Hou (D913788817@qq.com )

First Affiliated Hospital of Harbin Medical University

Haoxin Zhou

First Affiliated Hospital of Harbin Medical University

Research article

Keywords: ACS, elderly patients, ED visits, geriatric emergency patient, Enhanced Recovery after Surgery

Posted Date: May 19th, 2020

DOI: https://doi.org/10.21203/rs.3.rs-28535/v1

License: (c) This work is licensed under a Creative Commons Attribution 4.0 International License.

Read Full License 


\section{Abstract \\ Background}

There was a fast growth in the number and the formation of ED visits in China during the twenty-first century. As a result, engaging special medical model will be essential to decompressing the ED visits. To do this, it will be important to understand which specific aspects to focus interventions on for the greatest impact.

\section{Methods}

To characterize the emergency surgery patients who were seen and discharged from ED. Retrospective cohort study of hospitalized emergency surgery patients currently under the care from specialists presenting to an urban, university affiliated hospital between 01 January 2018 and 1 January 2019. This study will highlight some of the controversies and challenges and key lessons learned.

\section{Results}

During the study period there were 231,229 ED visits; 4,100 of these patients were admitted for ACS service. Multivariate analysis identified age $\geqq 65(p=0.023$; odds ratio, OR $=2.66)$, ACS model $(p=0.000$, $O R=0.18)$, ICU stay $(p=0.000, O R=118.73)$ as factors associated with in-hospital mortality. There was a increase in LOS between young and elderly postoperative patients when stratifying patients by age(11.67 \pm 9.48 vs $13.95 \pm 9.11 p<0.05$ ). we first came up with this concept of Fast Track Acute Care Surgery.

\section{Conclusions}

ED overcrowding is not just an ED problem. ED overcrowding is a systems problem requiring a systematic facility-wide multidisciplinary response. Continuous and high-quality surveillance data across China are needed to estimate the emerging FTACS model which used to deal with ED overcrowding. Trial registration: retrospectively registered

\section{Background}

Although emergency general surgery (EGS) and trauma patients have always been an integral part of surgical care, the field of acute care surgery (ACS) as a surgical specialty is a relatively new concept. ACS was initially proposed in 2005 as an innovative model of care to facilitate timely and high-quality EGS care along with trauma and surgical critical care ${ }^{[1]}$. Prior studies have demonstrated the ACS model can improve work flow and provide more effective delivery of care for patients with common urgent general surgery diagnoses without an increased rate of complications ${ }^{[2]}$. 
After much work over the past several years, the ACS model has entered the high-speed development period. However, there is a significant burden and unmet need for acute surgical conditions in low-income and middle-income countries(LMICs), especially country like China with large population base. According to Disease Control Priorities 3 released by the World Bank, 5 billion people in the world do not have access to safe surgical and anesthesia care, with a disproportionate 9 of 10 people in LMICs $^{[3]}$. Noncommunicable diseases including trauma and emergency surgery accounts for up to nearly one-third of the entire global burden of disease. In China, hospitals have noted an increased acuity of patients presenting to them. According to the "China Health Statistical Yearbook Compiled by the National Health and Family Planning Commission", the number of emergency department (ED) visits in Chinese hospitals has increased significantly from 107,805,396 in 2012 to 166,489,807 in 2017 over a 6-year period(http://www.yearbookchina.com/) (Fig. 1). Due to high volumes of emergency surgery, a special ACS model was developed at a Third Grade Class A hospital in Harbin,Heilongjiang province,China. The aim of this study was to describe the structure of the ACS service in our hospital, the epidemiology of ACS and and summarize experience to improve the efficiency of ACS service.

\section{Materials And Methods}

A retrospective, cross-sectional study was conducted on patients who visited the ED at the First Affiliated Hospital of Harbin Medical University which is a public hospital with 6496 beds located in Heilongjiang province between 1 January 2018 and 1 January 2019. The ACS surgeons at the First Affiliated Hospital of Harbin Medical University focus on 3 major specialty areas : trauma, general surgery and orthopedic surgery. The general surgery are subdivided by subspecialty expertise into colorectal, hepatic, breast, thyroid, oncology, gastrointestinal, vascular, pancreatic and endoscopic biliary surgery in our hospital. The orthopedic surgery are subdivided by subspecialty expertise into spine, joint, bone tumour, trauma and hand microsurgery. The bed capacities of the hospital in the general surgery unit, orthopedic surgery unit, ACS unit and intensive care unit are $768,455,111$ and 78 respectively.

The data were collected from electronic hospital medical records and patient ED charts, including the patients' demographic characteristics (age, gender and length of hospital stay), need for intensive care unit admission, presenting complain or ED diagnosis and outcome, including discharge and hospitalization rates and the date and in-hospital mortality. The primary analysis was conducted on the entire cohort of patients and included surgical priority (emergent and elective operations). Emergent cases were defined as an operation performed for an emergency medical condition that is expected to result in a severe adverse patient outcome in the absence of surgery (typically performed within 24 hours of admission). Elective operations are scheduled in advance, with an outpatient interval between decision to operate and actual operation.

Patients were assigned a diagnostic group based on their principal diagnosis utilizing the Clinical Classifications Software for International Classification of Diseases (ICD), 9th revision. ACS encompasses trauma, surgical critical care, and EGS. While the scope of trauma practice is well established, the last two components remain unclear. There is no unified definition of what constitutes an 
EGS patient and no easy way to identify them in existing databases, such as E-codes for trauma. So, patients in our study were divided into two groups: Trauma group and Non-Trauma group.

\section{Statistical analysis}

Data were summarized using descriptive statistics. Mean and standard deviation was presented for continuous variables, and frequencies and percentages for categorical variables. Independent $t$-tests were used to compare means and Pearson's Chi-square test for groups' comparison. Binary logistic regression analysis (backward model) was used to identify variables associated with in-hospital mortality. For continuous normally distributed data, the ANOVA test was used. Analysis was carried out using SPSS(Statistical Package for Social Science, IBM SPSS Statistics, Version 23 for Macintosh;IBM Corp.,Armonk,NY,USA). An alpha level of 0.05 was considered statistically significant.

\section{Results}

There were ten specialties ED represented in our hospital : surgical emergency, medical emergency, stomatological emergency, gynecological emergency, obstetric emergency, dematological emergency, neurosurgical emergency, neurology emergency, Ophthalmic emergency and pediatric emergency. During the study period there were 231,229 ED visits; 4,100 of these patients were admitted for ACS service ( Fig. 2). The largest volume of ED visits related to specialty services was in medical emergency, surgical emergency and pediatric emergency, respectively. Among the surgical emergency hospitalized patients, general surgery $(52.41 \% ; 2903 / 5539)$ and orthopedic surgery $(16.52 \% ; 915 / 5539)$ were the 2 most frequent sub-specialty and accounted for more than $68 \%$ of all sub-specialty.

A total of 5533 patients were selected for analysis. The overall mean age was $52.69 \pm 17.42$ years. When combined, a total number of 2689 (48.59\%) patients had an age range of 0-54 years, 2631 (47.55\%) patients were between the ages of 55 and 84 years, and 213 (3.85\%) patients were 85 years and older (Fig. 4 ). There were 1252 patients in Trauma group and 4281 patients in Non-Trauma group. Of the Trauma group, $60.78 \%$ were male and $39.22 \%$ were female, and for the non-Trauma group these were $56.57 \%$ and $43.42 \%$ respectively. There was a significantly higher lengths of LOS in Trauma group compared to the non-Trauma group $(11.76 \pm 13.38$ vs. $8.73 \pm 7.38, p=0.000)$. Two point ninety-nine percent of patients in the non-Trauma group and $2.32 \%$ of patients in the Trauma group died in hospital. Nevertheless, the above characteristics were not statistically different between the two groups, even if stratifying patients by age. There were significantly higher proportions of ICU stay in Trauma group compared with the non-Trauma group cohort(8.62\% v. $6.38 \%$, respectively, $\mathrm{P}=0.008)$. Of the Trauma group, $51.6 \%$ of patients received operative therapies, and for the non-Trauma group these were $48.05 \%$.

There was a trend for a greater proportion of patients in the Trauma group received emergency operative therapies when compared with the non-Trauma group (43.61\% vs. $26.77 \%$, respectively, $P=0.000$ ).

(Table 1) 
Multivariate analysis identified age $\geqq 65(p=0.023$; odds ratio, $O R=2.66)$, ACS model $(p=0.000, O R=$ $0.18)$, ICU stay $(p=0.000, O R=118.73)$ as factors associated with in-hospital mortality (Table 2$)$. Gender, trauma, emergency operation and length of stay (LOS) were not associated with increased mortality in the present study .

During the study period, we wondered if age factors were contributing to ED length of stay for surgical patients and thereby contributing to overall ED crowding. Therefore, we performed a study to determine the effect of all age ranges patients on overall ED length of stay. Further investigation revealed that most of the assumptions were not statistically significant. However, after stratifying patients by age, we found that there was a increase in LOS between young and elderly postoperative patients (11.67 \pm 9.48 vs 13.95 $\pm 9.11 \mathrm{p}<0.05$ ) (Table 3 ). Maybe the difference was not significant, whereas the elderly had higher rates of postoperative intergroup mortality in hospital (0.62 vs 8.56$)$.

\section{Discussion}

The ACS model was defined as a surgical team dedicated to the evaluation and management of urgent and emergent surgical consultations without having concurrent obligations related to the surgeons' elective practices. The traditional model of care for emergency surgery patients was an "on-call" system which was defined as having a rotating pool of general surgeons who would intermittently take call while continuing with their regularly scheduled clinical obligations ${ }^{[4]}$. Previous studies have demonstrated the improvement in injury-related mortality and length of stay in hospital systems that use this ACS model ${ }^{[5]}$. Furthermore, ACS also facilitates the logistics of caring for the acutely ill patient, maintains trauma surgeons' operative skills, and allows the elective surgeons to pursue an uninterrupted schedule. Despite these theoretical benefits to the hospital and surgical subspecialist, acute care surgeons often experience relatively some problems and dilemmas involved in exploding ED visits, elderly patients and discipline development ${ }^{[6]}$.In response to this challenge, a special ACS model was developed at a Third Grade Class A hospital in Harbin,Heilongjiang province,China. The key elements were:

* The impetus for the ACS target was the desire by speeding recovery to reduce ED overcrowding and hospital access block in the hope of improving both outcomes for patients and health service efficiency.

* ACS surgeons commit to being available for a 24-hour emergency duty omnibus diebus quaternis, and to lead a team consisting of a senior surgical resident, several junior residents, and medical students. Another teams were on call the other night. There are total four teams including 20 ACS surgeons in our ACS model.

* In this new model, the sub-specialty staff surgeons were available for a 24-hour emergency with ACS surgeons at the same time. It is worth noting that the sub-specialty staff surgeons who on emergency duty do not do elective surgery, but devote themselves to being available in a timely fashion to perform any emergency operation or provide emergency consultation. In our hospital, orthopedics and general 
surgery involve 14 sub-specialties, each of which is divided into multiple medical teams. Each medical team is responsible for one day's emergency treatment in turn.

* ACS surgeons are the dominant force in the treatment of emergency patients, and decide which patients need to be admitted to hospital and the departments in which they are admitted (such as ACS unit, general surgery unit, orthopedic surgery unit, etc.).

* In order to ensure the implementation of the ACS, we create a 24-h emergency outpatient surgery unit at our tertiary referral center. Emergency outpatient surgery differed from ambulatory surgery and emergency day-case surgery, as it implies either did not require hospitalization or a LOS less than $24 \mathrm{~h}$, which could include overnight hospitalization. All emergency outpatient procedures are performed by ACS surgeons.

* Setting targets for LOS of ED patients based on triage acuity, disease characteristics and monthly ED visits. ACS surgeons receive additional funds each month contingent on meeting ACS-specific improvements in achieving targets. This is called pay for performance: the "money".

* Other features include a major effort to improve patients' ward outflow by transfering some postoperative or alternate level of care patients to rehabilitation wards.

ED overcrowding has been defined as a situation in which demand for acute care exceeds the ability of physicians and nurses to provide timely quality care, which threatens patient health and fosters patient dissatisfaction ${ }^{[7]}$. Many factors can contribute to overcrowding : preemergency factors that drive demand for emergency services, patients frequently use ED for non-urgent conditions, size/capacity of the ED and its efficiency of processing, and flow out from the ED to the hospital wards ${ }^{[8]}$. Among these, we think that ED crowding is most commonly due to the boarding of inpatients lacking beds for extended periodsreducing or eliminating this should greatly improve ED efficiency. It's remarkable that saving 1 to 2 days per treated patient, hospital bed utilization will be reduced by $15 \%$.In many respects, our hospital has noted an increased acuity of ED visits presenting to us. There were 231,229 ED visits; 7,584 surgical emergency patients of these were hospitalization, corresponding to 21 surgical emergency patients were hospitalization every day; 4,100 of these patients were admitted for ACS service between 01 January 2018 and 1 January 2019. With these starling figures, there is no end in sight to today's health care crisis, and it probably has not reached its pinnacle.

Moreover, more than half of patients were over 55 (52.41\%), and 213 patients were 85 years and older in our study(Fig. 2 ). As is known to all, The World Health Organization (WHO) defines an elderly person as one who has a chronological age of 65 years or more. Life expectancy and the proportion of the elderly population are increasing worldwide. The population in China is also aging rapidly. According to the "2018 China Statistical Yearbook Compiled by National Bureau of Statistics of China (http://www.stats.gov.cn/)," people aged 65 and over accounted for $11.4 \%$ (158.31 million) of the total population in 2017 in China; by 2030, the population is predicted to be 1.46 billion, and $16 \%$ percent of Chinese citizens will be aged 65 and over ${ }^{[9]}$. Recent research has highlighted the importance of biological 
not just chronological age. Functional decline and chronic health conditions can appear earlier than 65 years, particularly among individuals who experience health disparities ${ }^{[10]}$. The increasing rate of serious injury among older adults represents a formidable challenge for geriatric care as elders fare worse in terms of risk of disability, hospital length of stay, and risk of mortality in comparison with younger individuals with similar injuries. For example, Sieling et al found that among the oldest old undergoing surgery for trauma, $8 \%$ of the patients experienced at least one medical complication, whereas $5 \%$ of the patients experienced multiple complications, including heart arrhythmias requiring stabilization, respiratory failure requiring ventilation, gastrointestinal tract bleeding, and urinary tract infections ${ }^{[11]}$. The issue of mortality is an important one in emergency surgery, especially in elderly patients. In our study, Multivariate analysis identified age $\geqq 65(p=0.023$; odds ratio, $O R=2.66)$ is the factor associated with inhospital mortality. The postoperative mortality increased with age is more pronounced, when stratifying patients by age. What is noteworthy is that there was also a increase in LOS between young and elderly postoperative patients $(11.67 \pm 9.48$ vs $13.95 \pm 9.11 p<0.05)$. In our ACS model, we reduce ED overcrowding and settle the problem of geriatric emergency patients by transfering some complicated diseases or the oldest old patients to the highly specialized surgical units.

As is known to all, surgeons are more often tending to "organ-specific" surgeon practices("breast surgeon";"pancreatic surgeon";"colorectal surgeon" ; "gastrointestinal surgeon"). Highly focused specialization in all aspects of medicine is accelerating as the complexities of care increase and the sophisticated demands of patients show no sign of abating. This has led a shift in focus from general surgery with broad spectrum of diseases and surgical techniques. Meanwhile medical specialization is increasingly being managed by minimal-invasive techniques ${ }^{[12]}$. Overall, the general surgery workforce has followed a trend of increased specialization. The era of the "omnipotent general surgeon" is to an end and in order to improve quality in specific areas many institutions dedicate most of their economic resources to highly specialized surgical units. In our hospital, the general surgery are subdivided by subspecialty expertise into colorectal, hepatic, breast, thyroid, oncology, gastrointestinal, vascular, pancreatic and endoscopic biliary surgery. The orthopedic surgery are subdivided by subspecialty expertise into spine, joint, bone tumour, trauma and hand microsurgery. Indubitability, some complicated or geriatric emergency patients will benefit more from the specialist treatment than ACS.

Though not currently well-described in the literature, many models across the world essentially added EGS services to existing trauma services, creating a hybrid trauma-EGS services. In China, ACS hospitals are concentrated in large academic referral centers. Although past studies have demonstrated improvements in efficacy at such institutions with ACS implementation, most of them appear to the hybrid model. In fact, in most of the world, general surgeons associated to other clinicians in a dedicated team to ACS patients, practice ACS by necessity and not based on specific training in the specialty. Focus on the facts that several models must be created based on patients' needs and fitted to the local/regional public health requirements. Most surgeons are familiar with the Chinese symbol for learning: Better master one than engage with ten. Compared with this hybrid model, the standard ACS model requires surgeons to acquire more skills, which often involve multiple specialties. This has resulted in heavy 
pressure on ACS surgeons and no formal ACS specialists in most countries. The danger ACS surgeons (and thereby trauma centers and systems) face is that they may attract few new recruits unless we provide a clear advantage and meaningful purpose to this career choice. So we first came up with this concept of Fast Track Acute Care Surgery (FTACS) through the implementation of this special model. we hope to determine the safety and fast of continuously receiving emergency patients at institutions with FTACS implementation.

Enhanced Recovery after Surgery (ERAS) programmes, also referred to as "fast track"(FT) perioperative care, are evidenced-based protocols designed to standardize and optimize perioperative care in order to reduce surgical trauma, perioperative physiological stress and organ dysfunction (metabolic, endocrine and inflammatory response as well as reduce protein catabolism) related to elective procedures ${ }^{[13]}$. Nowadays, a systematic review focusing on safety of ERAS for geriatric emergency patients was conducted by Paduraru $\mathrm{M}$ et al ${ }^{[14]}$. Their study demonstrated that ERAS can be safely applied to elderly and emergency patients with a reduction in postoperative complications, hospitalization and readmission rates. Patient compliance was also addressed in a number of the studies reviewed and ranged from 50 to $85 \%[15][16]$. Emergency surgery patients achieved an average level of compliance in comparison to elective surgical patients, again demonstrating that ERAS in emergency surgery is feasible. Certainly, one of the most challenging aspects of FTACS improvement is the selection of appropriate patient. In our new model, the initial vision is to triage some emergency patients and keep those patients suitable for rapid rehabilitation treatment in ACS. However, several challenges and opportunities associated with the implementation of this model have yet to be evaluated and overcome before a wider implementation. Moreover, more research is still needed in relation to identifying which elements of ERAS have greater impact on emergency surgery patients and whether independent impact plays a more significant role.

This study has a few limitations. First, it considered only one specialty care unit and ED at the same hospital, so similar studies in other settings might be conducted before forming broad conclusions. Other limitations include that this was a single center study at a large academic medical center in a metropolitan area with a large number of affiliated specialty services. The percentage of patients is likely higher than it would be in a community hospital. Finally, it is generally believed that both preoperative medical conditions and postoperative complications are important determinants for postoperative shortand long-term mortality of emergency patients. In our statistical analysis, we did not provide the details evidence indicates that intraoperative massive blood loss, blood transfusion, adverse cardiovascular events and total fluid volume are associated significantly with increased risks of postoperative mortality after emergency surgery. Our study does also not evaluate for differences in rates of operative, conservative, or interventional management, which could have large impacts on lengths of stay and outcomes.

\section{Conclusion}

The primary mission of the ACS service includes surgical critical care and the surgical management of acutely ill patients with a variety of conditions including trauma, burns,surgical critical care or an acute 
general surgical condition. In fact, ACS is an evolving concept which has not yet found its proper position in the surgical panorama. Recent studies have demonstrated that the "one-size-fits-all" model of ACS does not work. Ongoing research is needed to determine which patients and diseases are most effectively and efficiently cared for in ACS hospitals.

The department will continue to evaluate the effectiveness of this model and make changes based on additional data. The data in this article are encouraging for ACS surgeons and should lead other groups to develop their own ACS models and to prospectively collect and pool their data to validate this series.In conclusion, ED overcrowding is not just an ED problem. ED overcrowding is a systems problem requiring a systematic facility-wide multidisciplinary response.

\section{Abbreviations}

ACS

Acute care surgery

LMICs

low-income and middle-income countries

EGS

Emergency general surgery

AAST

the American Association for the Surgery of Trauma

ED

emergency department

ICD

International Classification of Diseases

LOS

length of stay

FTACS

Fast Track Acute Care Surgery

ERAS

Enhanced Recovery after Surgery

\section{Declarations}

\section{Availability of supporting data}

All data are fully available without restriction

\section{Ethical Approval and Consent to participate}

Not applicable 


\section{Consent for publication}

Not applicable

Conflict of interest All authors have no financial support and potential conflicts of interest for this work

Funding the national clinical key specialty construction project

Authors' contributions Not applicable

Acknowledgements Not applicable

\section{References}

1. Lyu, HG; Najjar, P; Havens, JM; Past, present, and future of Emergency General Surgery in the USA. [J].Acute Med Surg.2018,5(2):119-122

2. Bandy, NL; DeShields, SC; Cunningham, TD; et al.Statewide assessment of surgical outcomes and the acute care surgery model.[J].J Surg Res.2017,220(12):25-29

3. Merchant, Al; Walters, CB; Valenzuela, J; et al.Creating a Global Acute Care Surgery Fellowship to Meet International Need.[J].J Surg Educ.2017,74(5):780-786

4. Kurihara, H; Acute Care Surgery: A Necessity Across Europe. Are We Ready to Take the Lead? [J].Chirurgia (Bucur).2017,112(5):630-631

5. Ball, CG; Murphy, P; Verhoeff, K; et al.A 30-day prospective audit of all inpatient complications following acute care surgery: How well do we really perform?[J].Can J Surg.2020,63(2):E150-E154

6. Xu, D; Hou, L; Zhou, H; Challenges faced by acute care surgeons in China.[J].World J Emerg Surg.2019,14:16

7. Weiss, SJ; Derlet, R; Arndahl, J; et al.Estimating the degree of emergency department overcrowding in academic medical centers: results of the National ED Overcrowding Study (NEDOCS).[J].Acad Emerg Med.2004,11(1):38-50

8. Ovens, H; ED overcrowding: the Ontario approach.[J].Acad Emerg Med.2011,18(12):1242-5

9. Moran, A; Zhao, D; Gu, D; et al.The future impact of population growth and aging on coronary heart disease in China: projections from the Coronary Heart Disease Policy Model-China.[J].BMC Public Health.2008,8(27):394

10. Wang, C; Li, F; Wang, L; et al.The impact of population aging on medical expenses: A big data study based on the life table.[J].Biosci Trends.2018,11(6):619-631

11. Sieling, BA; Beem, $\mathrm{K} ;$ Hoffman, MT; et al.Trauma in nonagenarians and centenarians: review of 137 consecutive patients.[J].Am Surg.2004,70(9):793-6

12. Demirel, $A ; O z, M ; U l g e r, O ;$ The effect of minimal invasive techniques and physiotherapy on pain and disability in elderly: A retrospective study.[J].J Back Musculoskelet Rehabil.2019,32(1):63-70 
13. Brindle, M; Nelson, G; Lobo, DN; et al.Recommendations from the ERAS? Society for standards for the development of enhanced recovery after surgery guidelines.[J].BJS Open.2020,4(1):157-163

14. Paduraru, M; Ponchietti, L; Casas, IM; et al.Enhanced Recovery After Surgery (ERAS) - The Evidence in Geriatric Emergency Surgery: A Systematic Review.[J].Chirurgia (Bucur).2017,112(5):546-557

15. Roulin, D; Blanc, C; Muradbegovic, M; et al.Enhanced recovery pathway for urgent colectomy.[J].World J Surg.2014,38(8):2153-9

16. Wisely, JC; Barclay, KL; Effects of an Enhanced Recovery After Surgery programme on emergency surgical patients.[J].ANZ J Surg.2016,86(11):883-888

\section{Tables}

Table 1 Demographic and operative characteristics of the study population

\begin{tabular}{|c|c|c|c|c|}
\hline $\begin{array}{c}\text { Patients } \\
\text { Characteristics }\end{array}$ & $\begin{array}{l}\text { All patients } \\
n=5533(\%)\end{array}$ & $\begin{array}{l}\text { Non-Trauma patients } \\
\qquad \mathrm{n}=4281(\%)\end{array}$ & $\begin{array}{l}\text { Trauma Patients } \\
\text { n=1252(\%) }\end{array}$ & $P$-values \\
\hline Age(years) $\pm S D$ & $52.69 \pm 17.42$ & $55.73 \pm 17.48$ & $49.94 \pm 17.52$ & 0.456 \\
\hline $\begin{array}{l}\text { Sex } \\
\text { (male/female) }\end{array}$ & $3183(57.53) / 2350(42.47)$ & $2422(56.57) / 1859(43.42)$ & $761(60.78) / 491(39.22)$ & 0.008 \\
\hline LOS , (days) & $10.67 \pm 7.27$ & $8.73 \pm 7.38$ & $11.76 \pm 13.38$ & 0.000 \\
\hline ICU & $381(6.86)$ & $273(6.38)$ & $108(8.62)$ & 0.008 \\
\hline Mortality & $157(2.84)$ & 128(2.99) & $29(2.32)$ & 0.245 \\
\hline$\leqq$ Aged 54 & $28 / 2689(1.04)$ & $17 / 1922(0.88)$ & $11 / 767(1.43)$ & 0.211 \\
\hline Aged 55-64 & $24 / 1200(2.00)$ & $20 / 960$ (2.08) & $4 / 240$ (1.67) & 0.802 \\
\hline Aged 65-74y & $46 / 845(5.44)$ & $40 / 725$ (5.52) & $6 / 120(5.00)$ & 1.000 \\
\hline Aged 75-84y & $42 / 586$ (7.17) & $36 / 503$ (7.16) & 6/83 (7.23) & 1.000 \\
\hline$\geqq$ Aged $85 y$ & $17 / 213(7.98)$ & $15 / 171$ (8.77) & $2 / 42(4.76)$ & 0.535 \\
\hline Surgical operation & 2703(48.85) & 2057(48.05) & $646(51.60)$ & 0.029 \\
\hline \multicolumn{5}{|l|}{ Treatment method } \\
\hline Elective operation & 1011(18.27) & $911(21.28)$ & 100(7.99) & 0.000 \\
\hline Emergency operation & 1692(30.58) & 1146(26.77) & $546(43.61)$ & 0.000 \\
\hline Non-operation & $2830(51.14)$ & 2224(51.95) & $606(48.40)$ & 0.000 \\
\hline
\end{tabular}

Continuous variables are given as mean standard deviation, and categorical variables as number (percentage)

Table 2 Factors associated with in-hospital mortality (multivariate analysis) 


\begin{tabular}{lccc}
\hline Factors & \multicolumn{3}{c}{ Multivariate analysis (binary logistic regression) } \\
\cline { 2 - 4 } & $\boldsymbol{B}$ & $P$ value & AOR(95\% CI) \\
\hline Male & -0.462 & 0.085 & $0.63(0.41-0.96)$ \\
Age $\geq 65$ & 0.979 & 0.023 & $2.66(1.14-6.19)$ \\
\hline ACS & & & \\
\hline Trauma & -1.707 & 0.000 & $0.18(0.07-0.47)$ \\
\hline ICU stay & 0.089 & 0.751 & $1.09(0.63-1.89)$ \\
\hline Emergency operation & -0.571 & 0.091 & $0.56(0.29-1.09)$ \\
\hline LOS & -0.115 & 0.622 & $0.84(0.42-1.66)$ \\
\hline
\end{tabular}

Table 3 Interrelation analysis of LOS and age among postoperative patients

\begin{tabular}{|c|c|c|c|c|}
\hline & $\mathrm{n}$ & $\operatorname{LOS}\left(\overline{\mathbf{x}}_{ \pm \mathrm{s}}\right)$ & $F$ & $P$-values \\
\hline$\leqq$ Aged 54 & 1291 & $11.67 \pm 9.48$ & 3.622 & 0.006 \\
\hline Aged 55-64 & 622 & $12.97 \pm 10.64$ & & \\
\hline Aged 65-74y & 416 & $12.92 \pm 8.64$ & & \\
\hline Aged 75-84y & 257 & $13.08 \pm 7.97$ & & \\
\hline$\geqq$ Aged 85y & 78 & $13.95 \pm 9.11$ & & \\
\hline
\end{tabular}

* one-way ANOVA test

\section{Figures}


the number of ED visits in Chinese hospitals

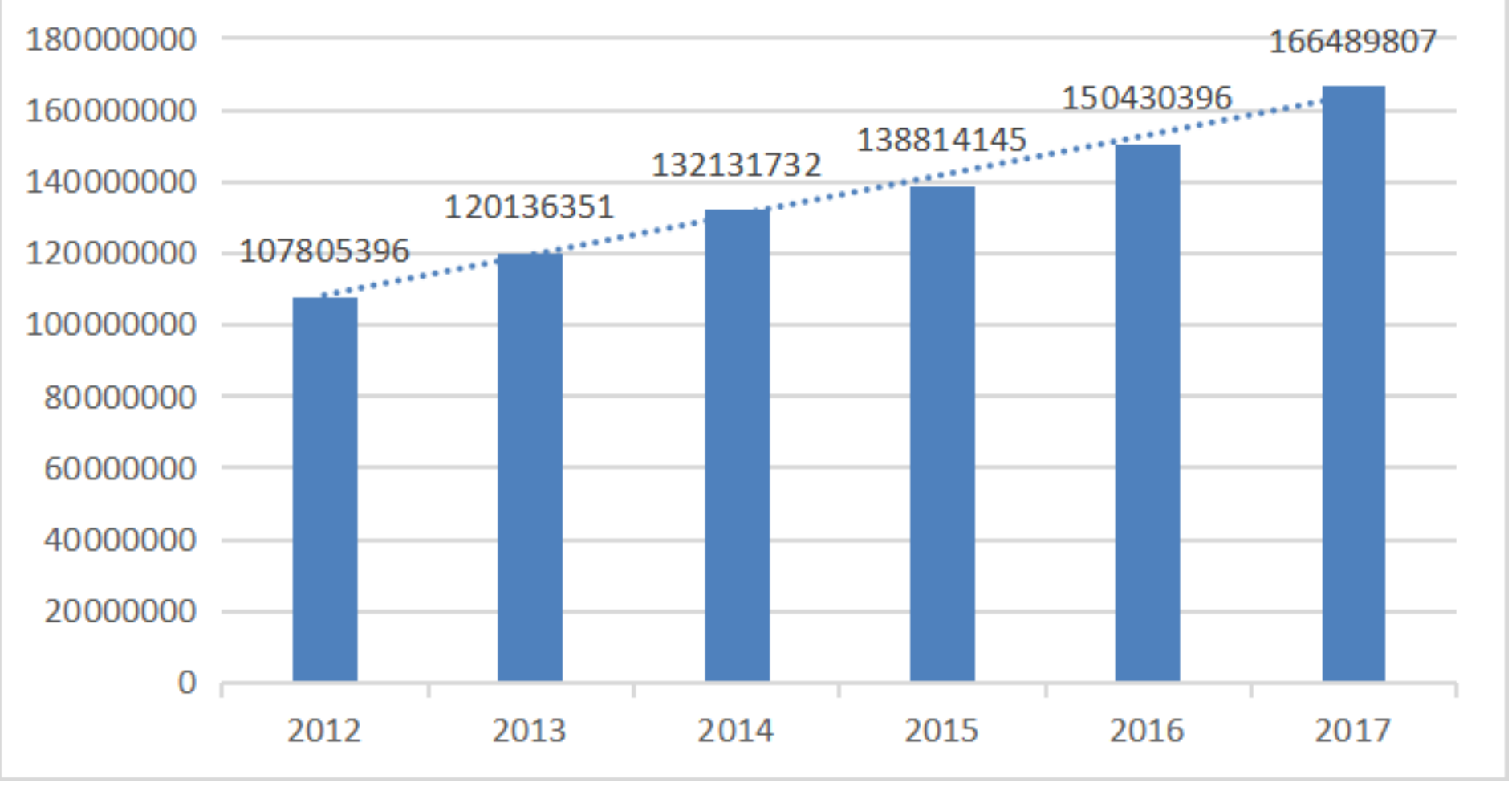

\section{Figure 1}

The number of ED visits in Chinese hospitals during the years 2012-2017 


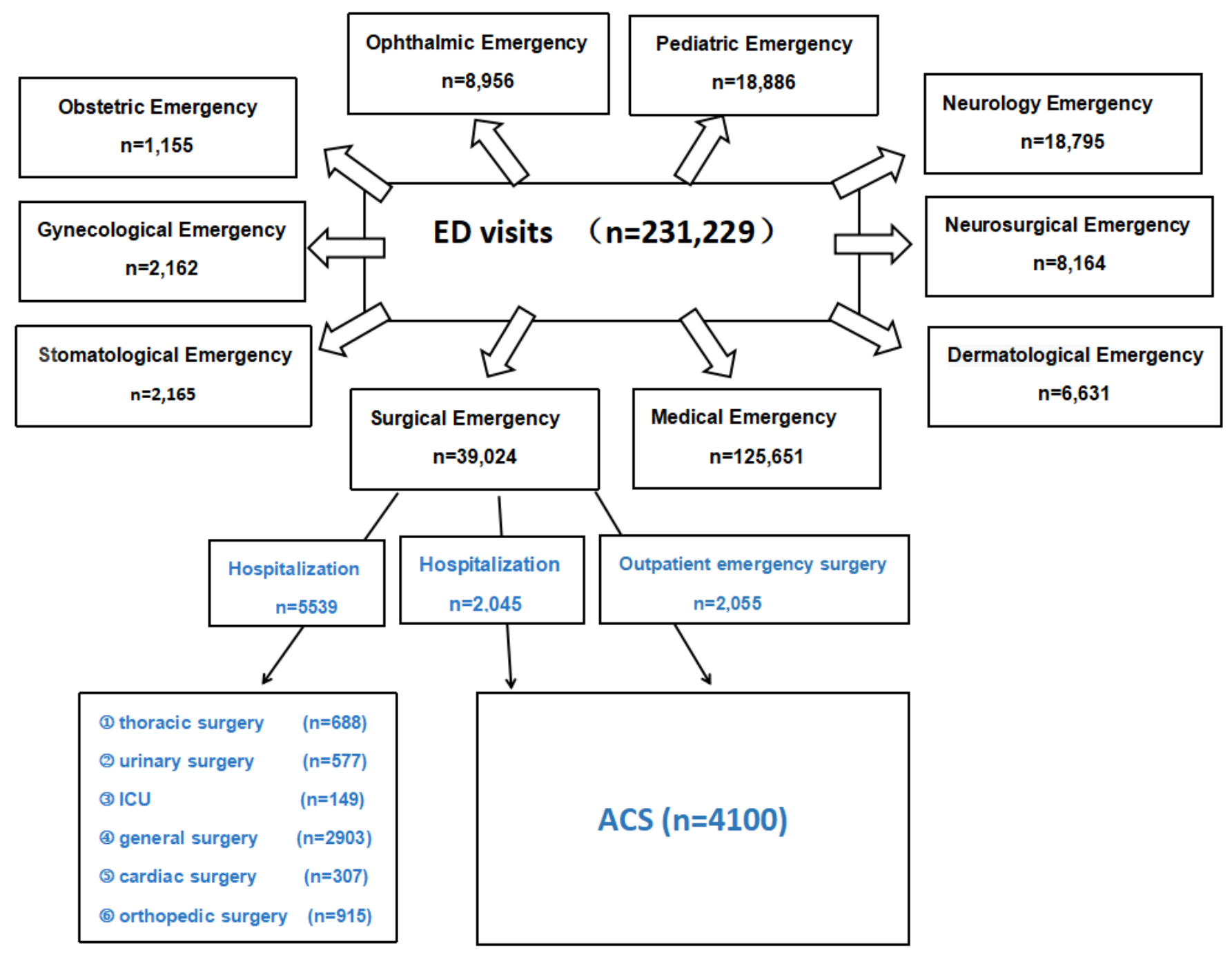

Figure 2

The population and distributing of ED visits at the First Affiliated Hospital of Harbin Medical University between 1 January 2018 and 1 January 2019. 


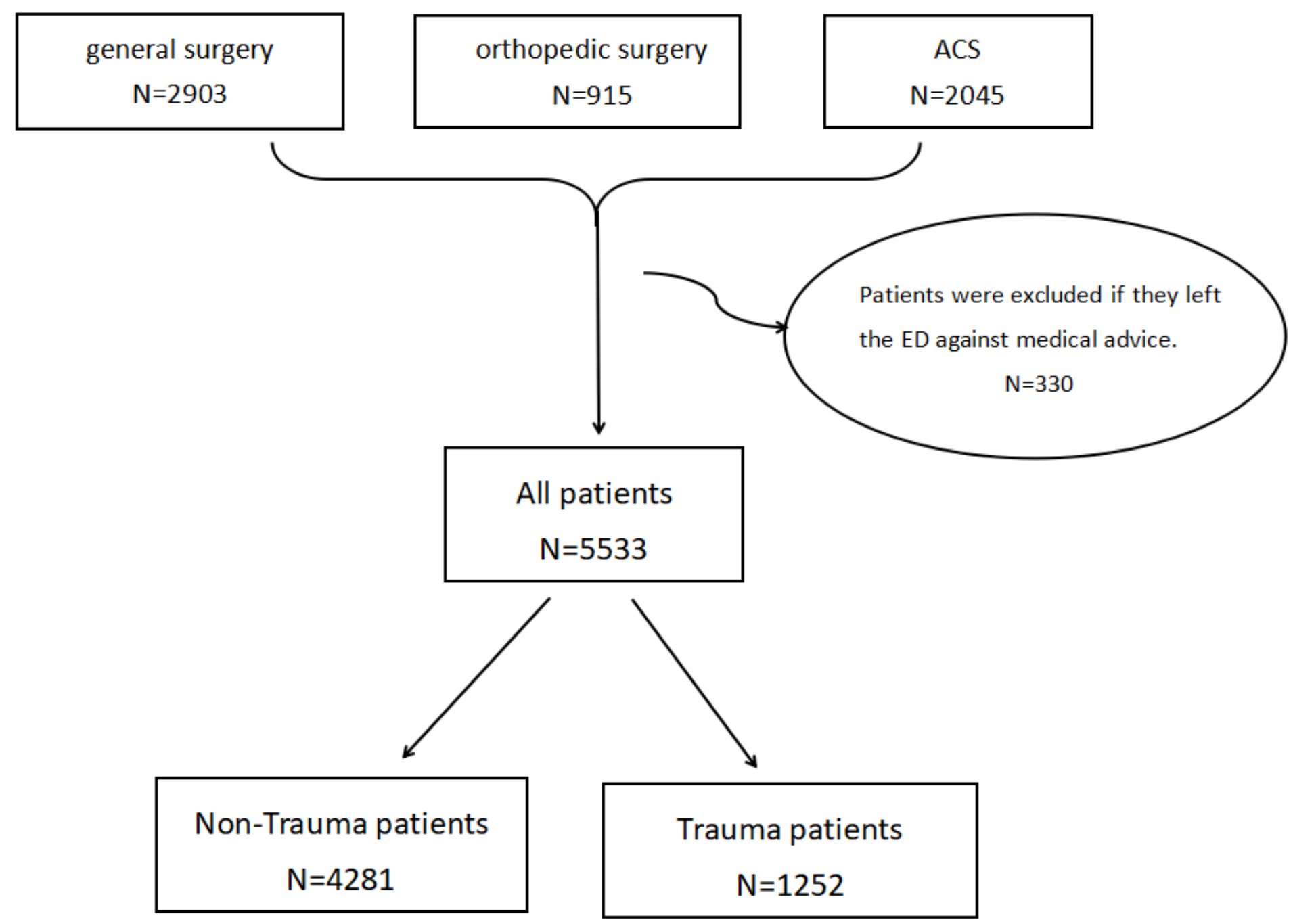

Figure 3

Patients selection process 


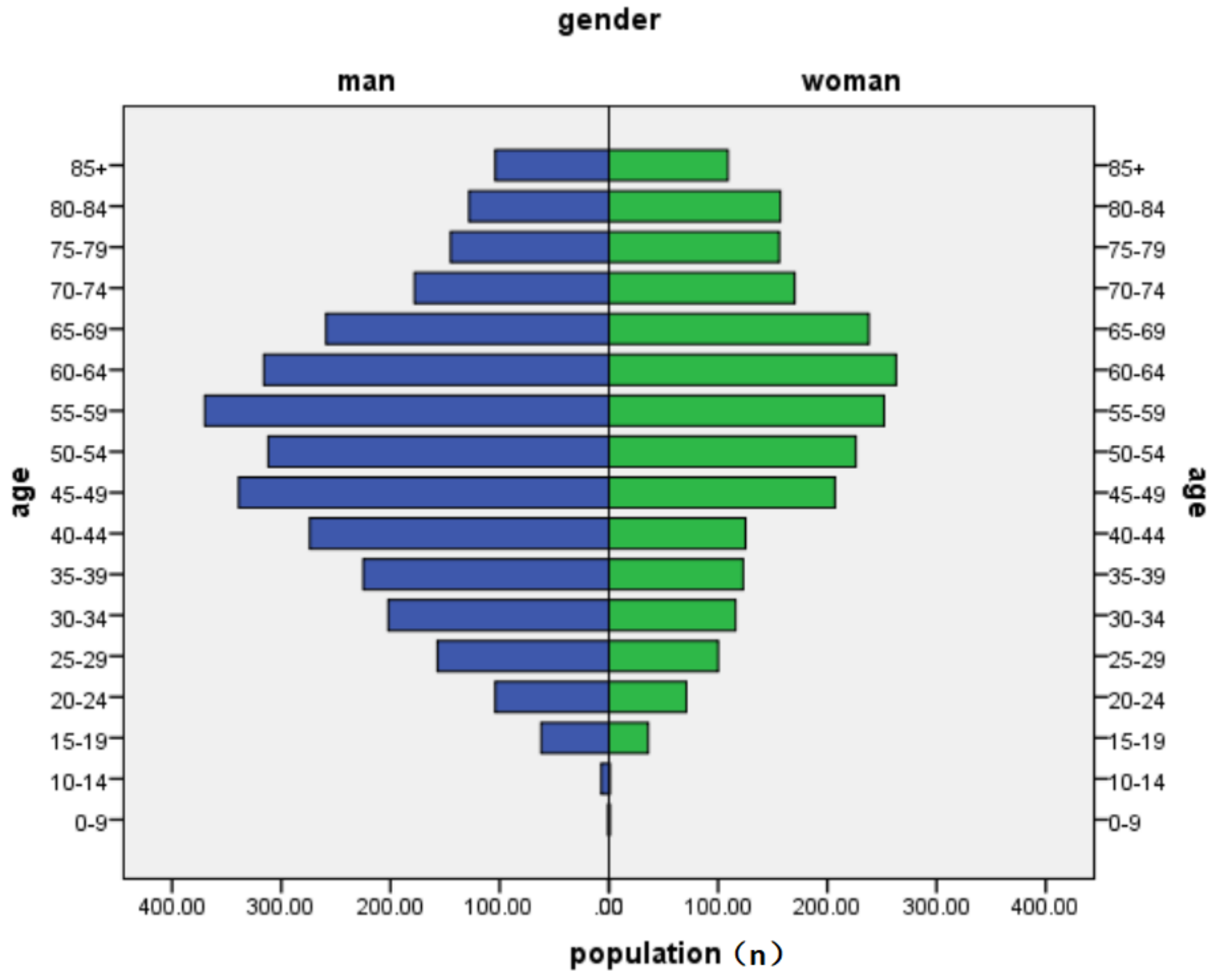

Figure 4

Age pyramid of 5533 patients were selected in our study 


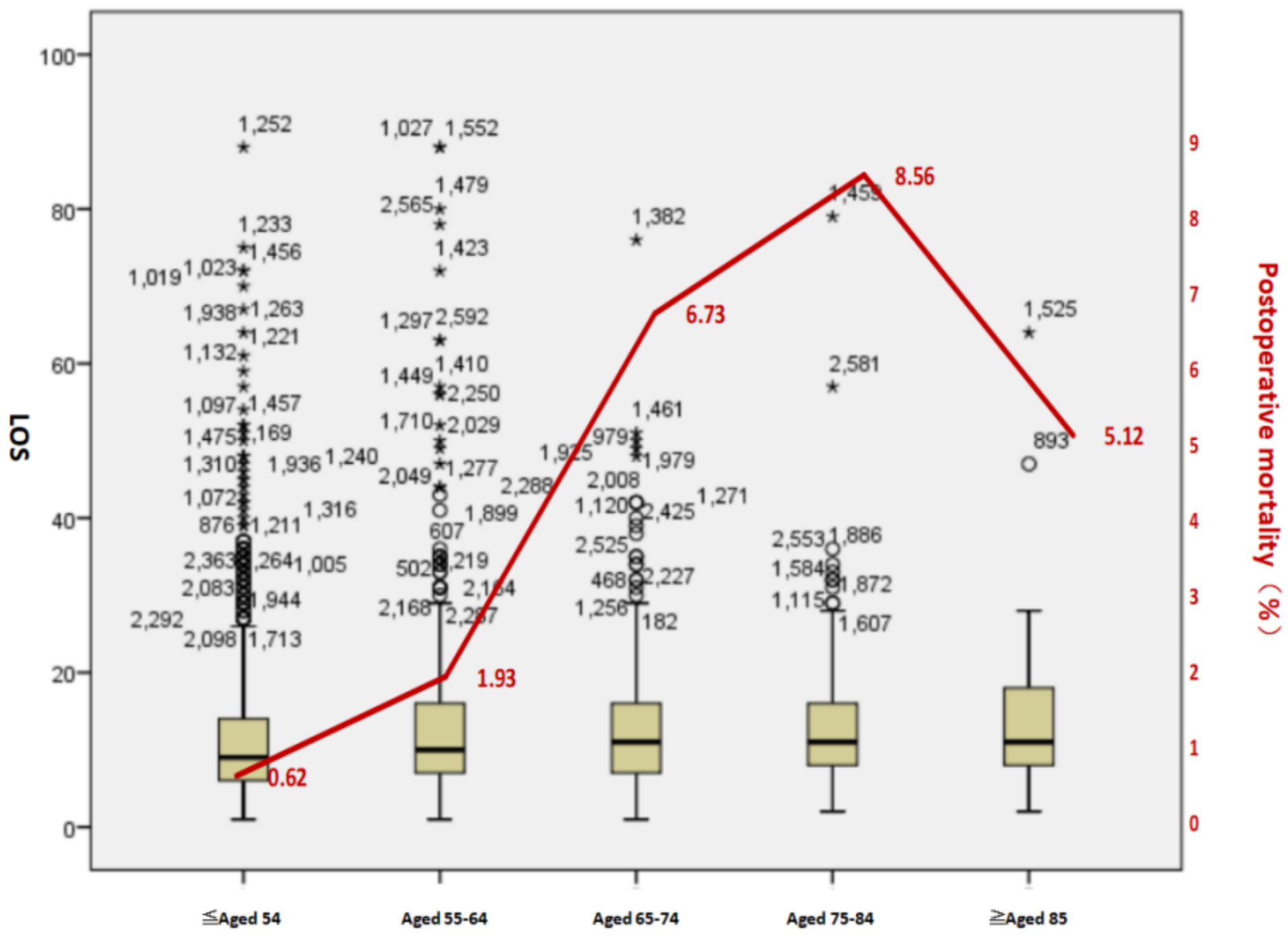

* The red line is the rates of postoperative intergroup mortality in hospital stratified by age

Figure 5

Stem-and-Leaf Plot :overall length of stay of patients stratified by age 